\title{
Anatomía de la madera de dos especies de Eugenia (Myrtaceae) de Quintana Roo, México
}

\author{
Wood anatomy of two species of Eugenia (Myrtaceae) \\ from Quintana Roo, Mexico
}

Silvia Rebollar-Domínguez ${ }^{1}$ y Nery Alicia Tapia-Torres 1

\begin{abstract}
RESUMEN
En México la familia Myrtaceae está representada por doce géneros que se distribuyen ampliamente en regiones templadas, tropicales y semiáridas. La importancia económica de esta familia se basa principalmente en la extracción de la madera (Eucalyptus spp.), el consumo de frutos (Psidium spp.) y la apicultura (Eugenia spp.), entre otros usos. En Quintana Roo se han registrado varios géneros de la familia, entre los cuales Eugenia, con varias especies está bien representado en la selva mediana subperennifolia. La madera de este género tiene usos locales importantes como postes y pilares en la construcción de casas rurales, así como para durmientes y son también valiosas especies melíferas. La estructura de su madera ha sido poco estudiada, por lo que en este trabajo se describe la anatomía de Eugenia capuli (Schlecht. \& Cham.) Berg. y Eugenia mayana Standley, especies colectadas en el ejido Cafetal Limones, Quintana Roo. Se realizó el estudio de sus características estéticas, anatómicas macroscópicas en tablillas de xiloteca y el microscópico en los tres cortes típicos y en material disociado; a los caracteres mensurables se les hizo un análisis estadístico y se determinaron con base en el valor de la media. Se incluyen en cada descripción los datos botánicos. La madera en ambas especies es de color castaño, con porosidad difusa, vasos con placa de perforación simple; el parénquima axial es en bandas y difuso; rayos heterogéneos tipos I, II, III y fibras libriformes. E. mayana se diferencia de E. capuli por presentar parénquima axial vasicéntrico, rayos triseriados y sus fibras presentan gomas.
\end{abstract}

PALABRAS CLAVE:

Eugenia capuli, Eugenia mayana, Quintana Roo, usos de la madera, Veracruz.

\begin{abstract}
The Myrtaceae family in Mexico is represented by twelve genera widely distributed in temperate, tropical and semiarid regions. The family has economic importance due mainly to the extraction of wood (from Eucalyptus spp.), of fruit for human use (Psidium spp.) and to the practice of apiculture (in Eugenia spp.), among other uses. Several genera representing the family have been recorded in Quintana Roo, among which Eugenia, with several species, is well represented in the tropical rainforest. Wood of this genus has important local uses: poles and pillars used in the construction of rural houses, it has also been used to build railroad (sleepers). Valuable melifer species have also been found. Its wood structure has been poorly studied; therefore this paper describes the anatomy of Eugenia capuli (Schlecht. \& Cham.) Berg. and of Eugenia mayana Standley, wich were collected in the common land (ejido) Cafetal Limones, Quintana Roo. The anatomical descriptions, have produced esthetic, macroscopic and microscopic studies using the three typical cuts and dissociated material. A statistical analysis was done using the measurable characters which were determined based on the value of the mean. Botanical data was included in each description. In both species, the wood is light brown, with diffuse-porous and the vessels have simple perforation plates; the axial parenchyma comes in stripes and it is diffused; rays are heterogeneous type I, II, III and libriform fibres. E. mayana is different from E. capuli in that it has axial parenchyma vasicentric, triseriate rays and its fibres have gums.
\end{abstract} mento de Biología. Av. San Rafael Atlixco \# 186. Col. Vicentina Iztapalapa D.F. C.P 09340. sired@xanum.uam.mx, aliciatt@gmail.com. 
KEY WORDS:

Eugenia capuli, Eugenia mayana, Quintana Roo, end-uses, Veracruz.

\section{INTRODUCCIÓN}

En Quintana Roo el manejo de las selvas ha girado en torno a dos actividades principales, una es la agricultura de autoconsumo y otra el aprovechamiento de los recursos no maderables y maderables, este último es un importante recurso ecológico y económico, ya que las selvas ofrecen una diversidad de especies arbóreas no conocidas en su anatomía, consideradas corrientes o duras, que tienen usos locales importantes para construcción rural, aunque pueden ser consideradas como alternativas para otros usos (Merino 1992; Rebollar et al., 1993; Rebollar y Quintanar, 1998; 2000).

La familia Myrtaceae es un ejemplo de lo dicho, en México esta familia tiene registrados 12 géneros y 124 especies (Monroy-Ortiz y Monroy, 2006), que están ampliamente distribuidos en regiones templadas, tropicales y semiáridas. Se reconoce que tienen gran importancia económica al encontrarse en ella plantas de interés y utilidad como maderables: Calyptranthes pallens Griseb., Myrcianthes fragans (Swartz) McVaugh, Eucalyptus camaldulensis Dehnh., entre otras; por su fruto comestible: Psidium sartorianum (Berg.) Ndzu., Eugenia biflora (L.) DC.; para la obtención de especias, aceites esenciales y condimentos: Pimenta dioica (L.) Merr., Sizygium aromaticum L.; en producción de miel: Eugenia jambos L.; como medicinal: Eucalyptus globulus Labill, Psidium guajava L. y ornamental: Sizygium jambos (L.) Alston, Psidium cattleianum Sabine (Terán y Rasmussen 1994; Cabrera et al., 2001; Arellano et al., 2003; Yáñez, 2004; Anderson et al., 2005; Monroy-Ortiz y Monroy, 2006).
Eugenia es un género que se distribuye en América, Asia, África y Australia (Record y Hess, 1949; Johnson y Briggs, 1984). En México, está representado por 80 especies, que se distribuyen ampliamente en las regiones tropicales y tienen importancia económica medicinal, maderable y comestible (Monroy-Ortiz y Monroy, 2006). De acuerdo con Arellano et al. (2003), en la península de Yucatán el género Eugenia tiene 16 especies, de las cuales 15 se consideran melíferas y cinco son utilizadas como combustible (leña).

Estudios relacionados con la anatomía de la madera de este género son los realizados por Barajas-Morales et al. (1997) y Ángeles (1981), en las especies: E. acapulcensis Steud. y E. origanoides Berg., respectivamente; ambas pertenecientes a una selva alta perennifolia de una región de Los Tuxtlas en Veracruz, México; otro es de la especie brasileña $E$. involucrate DC., descrita por Marchiori (1984). En este trabajo se presenta la anatomía de la madera de E. capuli y E. mayana, cuya madera se colectó en el ejido Cafetal Limones, Quintana Roo localidad con intensa actividad forestal, donde están bien representadas en la selva mediana subperennifolia y tienen importancia en la localidad por ser utilizadas para postes y pilares en la construcción de casas rurales y para la elaboración de durmientes de acuerdo con la dirección técnica forestal de la Organización de Ejidos Productores Forestales de la Zona Maya, S.C. (OEPFZM, S.C., 2000).

\section{OBJETIVO}

El objetivo de este trabajo es describir las características anatómicas de la madera de Eugenia capuli y Eugenia mayana, especies utilizadas en construcción rural. 


\section{ZONA DE ESTUDIO}

El ejido Cafetal Limones se ubica entre los paralelos $19^{\circ} 01^{\prime}$ y $19^{\circ} 27^{\prime}$ de latitud norte y en los meridianos $88^{\circ} 06^{\prime}$ y $88^{\circ} 30^{\prime}$ de longitud oeste, en el municipio de Othón $\mathrm{P}$.
Blanco, Quintana Roo (Figura 1). Tiene una altitud de 25 msnm y una extensión ejidal de 20681 hectáreas, con una población de 1 961 personas registradas en el II conteo de población (INEGI, 2005).

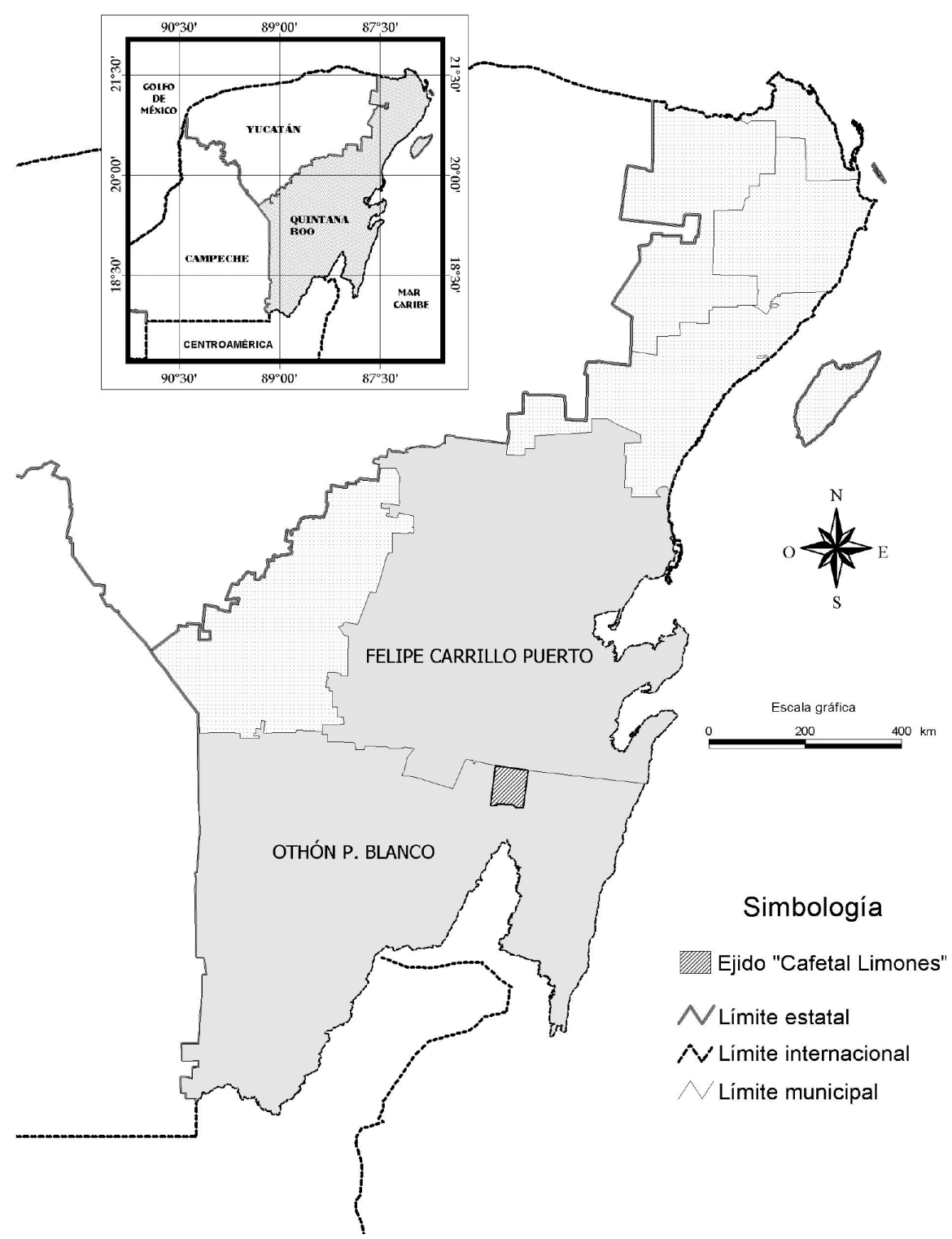

Figura 1. Ubicación de la zona de estudio. 
Cafetal Limones perteneció a la Organización de Ejidos Productores Forestales de la Zona Maya, S.C. (OEPFZM, S.C.), con la cual su gente ha trabajado en diversos proyectos que le ha permitido tener asesoría técnica y capacitación en sus actividades productivas. La actividad económica más importante es la forestal, con la producción de maderas preciosas y otras tropicales duras y suaves; y otras alternas son la siembra de chiles jalapeños, maíz y frutales, algunos ejidatarios tienen ranchos donde se puede encontrar ganadería y agricultura de temporal, así como la producción y comercialización de miel de abeja a granel (OEPFZM, S.C., 2000).

\section{METODOLOGÍA}

La madera de E. capuli y E. mayana, provino de árboles sanos, maduros, de fustes rectos y uno por especie, con alturas de $12 \mathrm{~m}$ y diámetros normales de $20 \mathrm{~cm}$ y $25 \mathrm{~cm}$, respectivamente. La colecta se hizo en una selva mediana subperennifolia perteneciente al ejido Cafetal Limones en el municipio de Othón P. Blanco, Quintana Roo, de acuerdo a las especificaciones de Ramos y Díaz (1981).

La selección de las trozas y muestreo para obtener el material para los estudios de sus características estéticas, macroscópicas se realizó en tablillas de xiloteca de $15 \times 7 \times 1 \mathrm{~cm}$ y las microscópicas en probetas de $2 \times 2 \mathrm{~cm}$ ablandadas siguiendo las técnicas apropiadas para maderas duras, para la elaboración de preparaciones fijas con los tres cortes típicos de la madera usando un micrótomo de deslizamiento. Los cortes se tiñeron en safranina acuosa a $1 \%$, se deshidrataron y se montaron con resina sintética $y$, de acuerdo al método de Jeffrey se obtuvo el material disociado
(Rebollar et al., 1987; 1993). La denominación de los caracteres macroscópicos y estéticos se clasificó de acuerdo con Tortorelli (1956) y el color se definió con las tablas de Munsell (1954).

La nomenclatura de los caracteres microscópicos se presenta de acuerdo con IAWA Committee (1989) y para los rayos se uso la clasificación de Kribs (1968); a los caracteres mensurables se les hizo un análisis estadístico con un error de muestreo de $5 \%$, donde se considera el valor de la media para su clasificación de acuerdo con Chattaway (1932) y con IAWA Committee (1937; 1939). El valor para poros se da en número por milímetro cuadrado $\left(\mathrm{mm}^{2}\right)$; el número de rayos por milímetro lineal $(\mathrm{mm})$ y las dimensiones de los demás elementos constitutivos en micrómetros $(\mu \mathrm{m})$.

Se presenta la descripción botánica y la sinonimia de las dos especies de acuerdo con Cabrera et al. (2001) y McVaugh (1963); la información de los nombres comunes y usos locales se obtuvieron por comunicación directa con los campesinos mayas de la localidad de colecta, cotejándola y ampliándola de acuerdo con Barrera et al. (1976), Sosa et al. (1985), Terán y Rasmussen (1994), Arellano et al. (2003) y Anderson et al. (2005). La distribución en México se consultó en: Johnson y Briggs 1984; Ibarra-Manríquez et al., 1995; ValienteBanuet et al., 1995; Gutiérrez, 2000; 2004; Martínez et al., 2001; Cartujano et al., 2002; Arellano et al., 2003; Castillo et al., 2003; Yáñez, 2004; Monroy-Ortiz y Monroy, 2006 y Padilla-Velarde et al., 2006. El trabajo se ilustra con el mapa de la zona de estudio, fotografías de la estructura anatómica y los resultados de las descripciones microscópicas se presentan en tablas. 


\section{RESULTADOS}

\section{Eugenia capuli (Schlecht. \& Cham.) Berg}

Sinonimia: Myrtus capuli Schlecht. \& Cham., Eugenia schiedeana Schlecht. E. capuli $\mu$ micrantha Berg., E. capuli $b$ macroterantha Berg., E. capuli g rigida Berg., E. contrerasii Lundell, Wrightia, E. tenuisima Lundell, Wrightia.

Familia: Myrtaceae.

Nombres comunes en la Península de Yucatán: Pichiche', guayabillo.

Distribución en México: Colima, Chiapas, Querétaro, Península de Yucatán, Tamaulipas y Veracruz.

Descripción botánica: Árbol de 6 a 12 $\mathrm{m}$ de altura, conserva su follaje durante la temporada seca del año, el tallo de $20 \mathrm{~cm}$ de diámetro, ramificado desde su base, la corteza amarillenta, escamosa. Las hojas son opuestas, simples, con el ápice acuminado y el margen entero, glabras y con numerosas glándulas translúcidas; en las hojas de más edad suele ser evidente un punto oscuro en la cara abaxial. La inflorescencia es de racimos cortos dispuestos en las ramas maduras, los pétalos blancos, ovario bilocular y óvulos de 8 a 10 en cada lóculo. Los frutos son drupas esféricas, de $5 \mathrm{~mm}$ de diámetro, coronados por el cáliz persistente y amarillo brillante al madurar.

\section{Características anatómicas de la madera:}

\section{Estéticas}

La madera presenta diferencia entre la albura y el duramen, la albura es castaño claro (10YR 6/4) y el duramen grisáceo (10YR 4/3). Tiene olor y sabor aceitoso, veteado suave, brillo mediano, textura fina e hilo recto.

\section{Macroscópicas}

Los elementos constitutivos son visibles con lupa (6x); las zonas de crecimiento están marcadas por fibras y poros solitarios.

\section{Microscópicas}

Los poros son de distribución difusa, la mayoría solitarios y múltiples radiales de 2 a 5 , de contorno oval y redondo, son muy numerosos $(\bar{x}=60)$, de diámetro tangencial moderadamente pequeño $(\bar{x}=$ $66 \mu \mathrm{m}$ ) (Figuras 2a y 2b).

Los elementos de vaso son medianos ( $\bar{x}=$ $498 \mu \mathrm{m})$, presentan puntuaciones areoladas alternas y placa de perforación simple, con presencia de colas y gomas. El parénquima axial es en bandas de 2 a 4 hileras de células y difuso escaso, presenta cristales romboidales y gomas. Se encuentran idioblastos con cristales romboidales. Los rayos son uniseriados y biseriados, pocos $(\bar{x}=4)$, heterogéneos tipo II y III, el tipo II tiene células procumbentes de 4 a 12 hileras con márgenes de células erectas de 1 a 3 hileras y el tipo III tiene un cuerpo de procumbentes de 2 a 11 hileras y margen de células cuadradas, son extremadamente bajos $(\bar{x}=238 \mu \mathrm{m})$ y muy finos $(\bar{x}=21 \mu \mathrm{m})$ con gomas. Los más abundantes son los biseriados. Las fibras son de tipo libriforme de longitud mediana $(\bar{x}=1095 \mu \mathrm{m})$, diámetro mediano $(\bar{x}=20$ $\mu \mathrm{m})$ y con paredes gruesas $(\bar{x}=10 \mu \mathrm{m})$ (Figuras 2c y 2d, Tabla 1).

\section{Usos locales}

La madera es dura por lo que es usada para la construcción rural. 


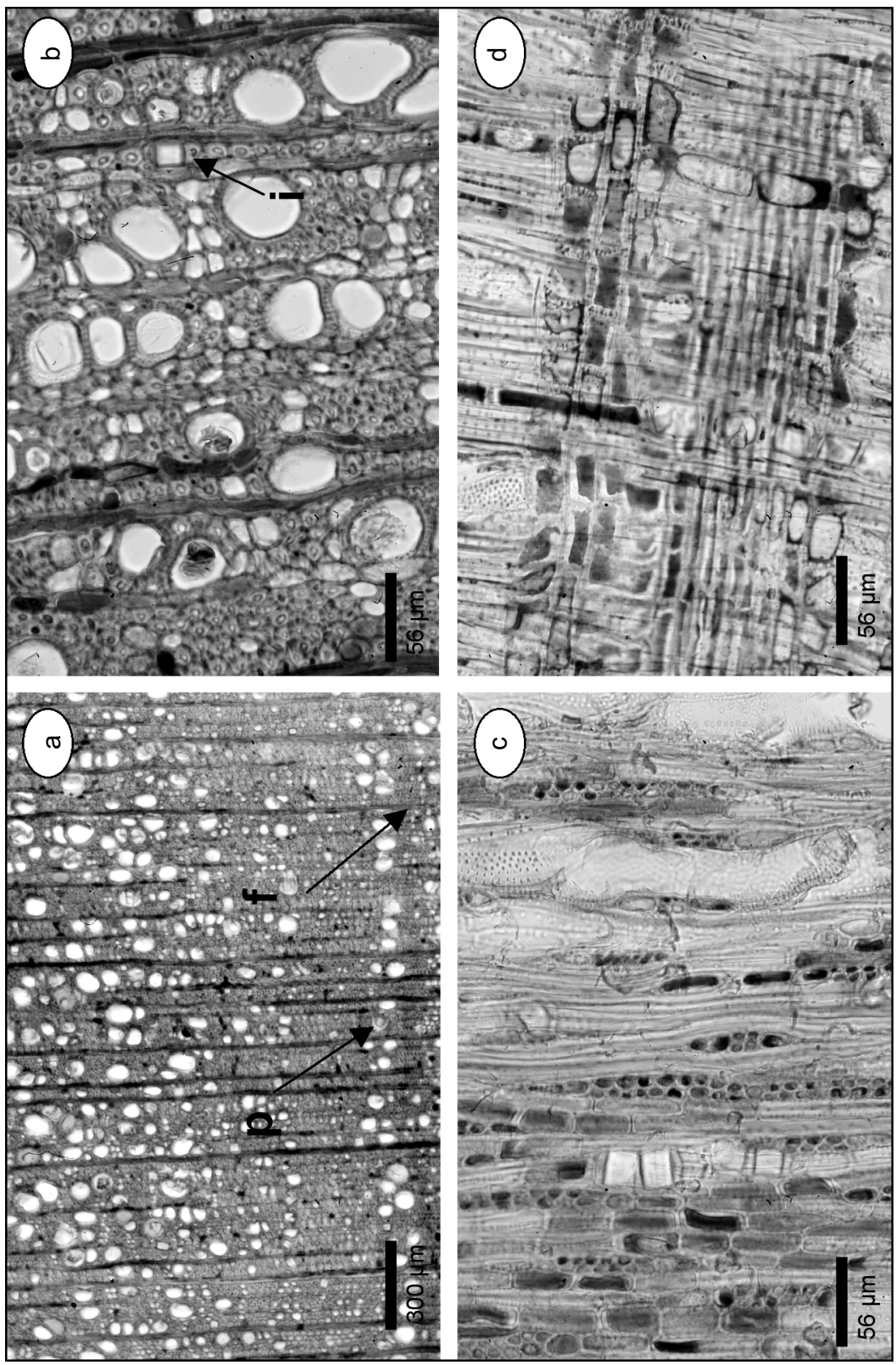

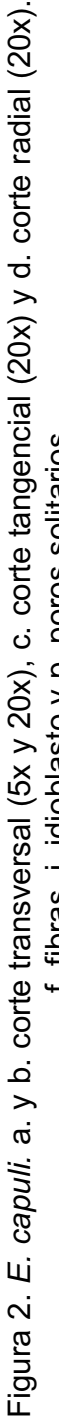




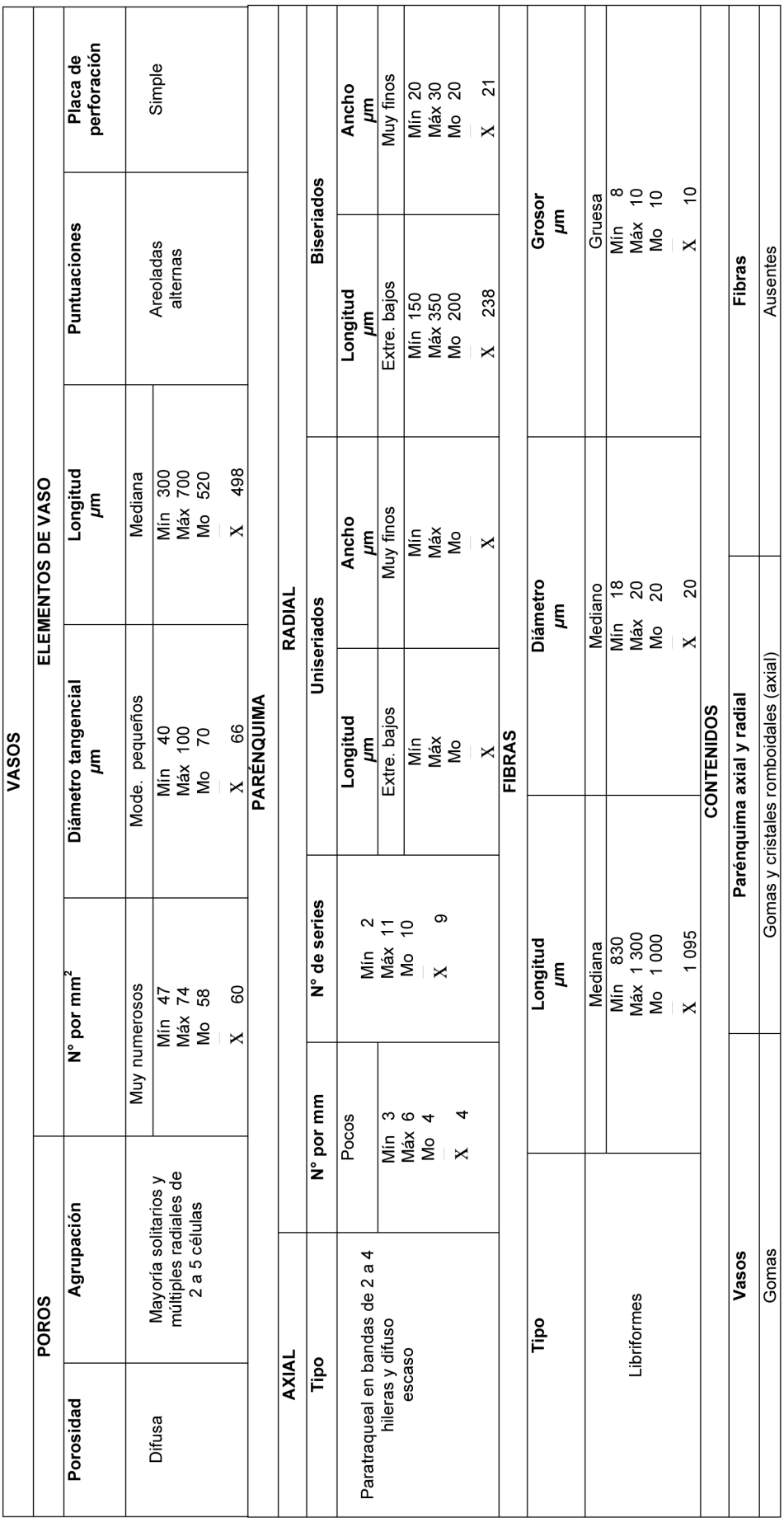




\section{Eugenia mayana Standley}

Sinonimia: E. buxifolia (Sw.) Willd., Myrtus buxifolia Sw., E. mayana Standl.

Familia: Myrtaceae.

Nombres comunes en la Península de Yucatán: Sakloob, xhil nich', xjirnich, jirimich, guayabillo.

Distribución en México: Chiapas y Península de Yucatán.

Descripción botánica: Árbol de 6 a 12 $\mathrm{m}$ de altura, conserva su follaje durante la temporada seca del año, el tallo de $25 \mathrm{~cm}$ de diámetro. Las hojas son ovadas o elípticas, ápice acuminado y el margen entero, glabras y glándulas translúcidas.

La inflorescencia en racimos cortos dispuestos en las ramas maduras, los pétalos glabros, ovario bilocular y óvulos de 5 a 6 en cada lóculo; la flor terminal generalmente es abortada. Los frutos son drupas esféricas, de $5 \mathrm{~mm}$ de diámetro, color negro.

\section{Características anatómicas de la madera:}

\section{Estéticas}

La madera presenta diferencia de color entre la albura y el duramen, la albura es castaño grisáceo (7.5 YR 6/4), el duramen es castaño oscuro (7.5 YR 5/4), con vetas oscuras (7.5 YR 3/0). Tiene olor y sabor aceitoso, veteado suave, brillo mediano, textura fina e hilo recto.

\section{Macroscópicas}

Los elementos constitutivos son visibles con lupa (6x); las zonas de crecimiento están marcadas por las fibras.

\section{Microscópicas}

Los poros son de distribución difusa, la mayoría solitarios, múltiples radiales de 2 a 4 , de contorno oval y redondo, muy numerosos $(\bar{x}=55)$, de diámetro tangencial moderadamente pequeño $(\bar{x}=65 \mu \mathrm{m})$ (Figuras 3a y 3b).

Los elementos de vaso son medianos $(\bar{x}=571 \mu \mathrm{m})$, presentan puntuaciones areoladas alternas y placa de perforación simple. El parénquima axial es en bandas de 2 a 4 hileras, vasicéntrico y difuso con gomas y cristales romboidales. Los rayos son uniseriados, biseriados y triseriados, pocos $(\bar{x}=3)$, homogéneos escasos, heterogéneos tipo I y III, el tipo I tiene células procumbentes de 4 a 9 hileras con erectas de 1 a 3 en los márgenes y el tipo III tiene células procumbentes de 4 a 10 hileras y un margen de células cuadradas, son extremadamente bajos $(\bar{x}=200 \mu \mathrm{m})$ y muy finos $(\bar{x}=20 \mu \mathrm{m})$, con gomas. Los más abundantes son los biseriados. Las fibras son de tipo libriforme, de longitud mediana $(\bar{x}=1327 \mu \mathrm{m})$ y diámetro mediano $(\bar{x}=20 \mu \mathrm{m})$ con paredes gruesas $(\bar{x}=5 \mu \mathrm{m})$, con gomas (Figura 3 y Tabla 2).

\section{Usos locales}

La madera es considerada dura por lo que es usada para construcción rural. 


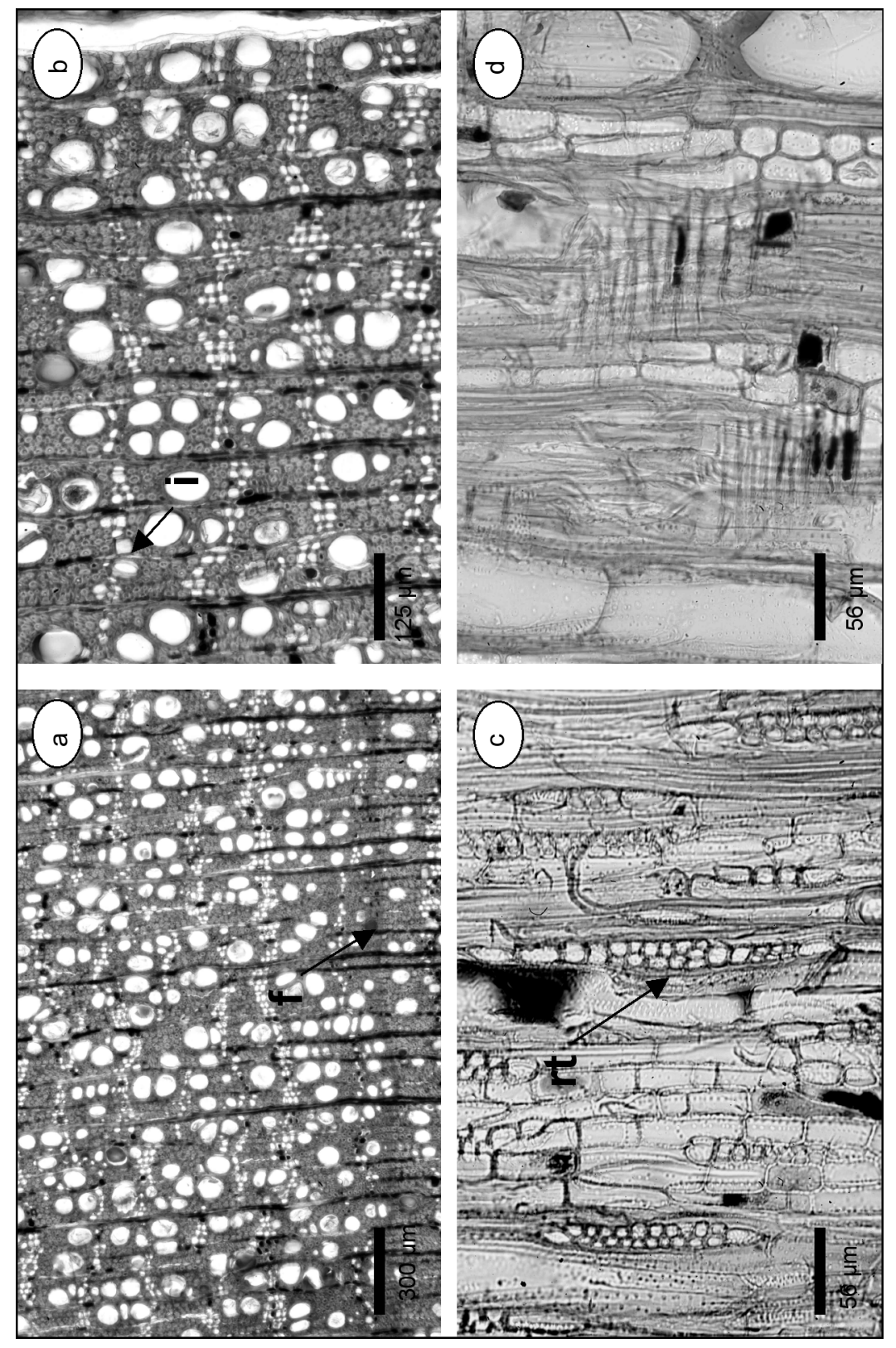

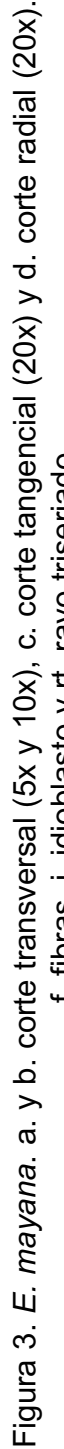




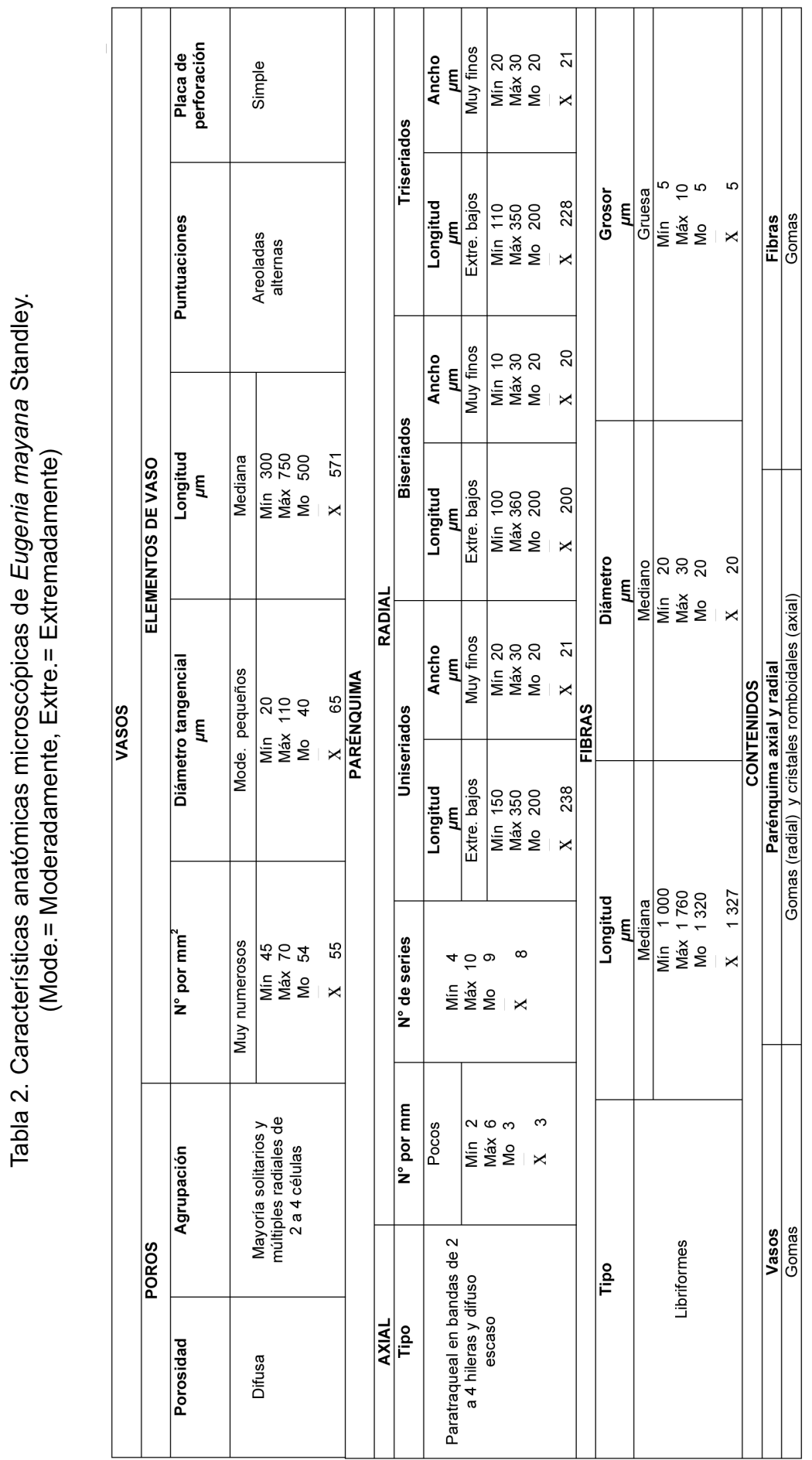




\section{DISCUSIÓN Y CONCLUSIONES}

La madera de las dos especies estudiadas presenta diferencia de color entre la albura y el duramen, para E. capuli es de tonalidades de castaño claro y grisáceo, E. mayana las tiene de color castaño grisáceo y castaño oscuro respectivamente, para la última especie se marcan vetas oscuras; la diferencia en color entre la albura y el duramen de ambas especies le proporciona a la madera un valor estético, por lo que se pueden usar en decoración. Comparten también olor y sabor aceitoso, brillo mediano, veteado suave, hilo recto $y$ textura fina. Las zonas de crecimiento están claramente marcadas por las fibras y en el caso de E. capuli además por la presencia de poros solitarios (Figura 2a).

En la estructura microscópica las dos especies tienen porosidad difusa, la mayoría de los poros son solitarios de contorno oval o circular y algunos múltiples radiales, muy numerosos, de diámetro moderadamente pequeño, longitud mediana con puntuaciones areoladas alternas y placa de perforación simple.

El parénquima axial es en bandas de 2 a 4 hileras de células y difuso, para las dos especies y E. mayana presenta también de tipo vasicéntrico. Los rayos de las dos especies, son pocos, moderadamente bajos y muy finos, siendo más abundantes los biseriados. E. capuli presenta rayos de tipo heterogéneos II y III, mientras que E. mayana tiene homogéneos escasos; heterogéneos de tipo I y III y presenta rayos triseriados (Figura 3c).

Las fibras son de tipo libriforme, de longitud y diámetro mediano y paredes gruesas. En el caso de E. mayana presentan gomas. Las características anatómicas de las dos especies de Quintana Roo aquí presentadas en compara- ción con E. origanoides y E. involucrate del estado de Veracruz, las cuales presentan datos similares es que en las últimas dos no se reportan olor ni sabor, el hilo es ligeramente entrecruzado y las zonas de crecimiento son inconspicuas. Los poros se encuentran por $\mathrm{mm}^{2}$ en menor número $(\bar{x}=13)$ que las de Quintana Roo ( $\bar{x}=55$ y $\bar{x}=60$ ), y el diámetro de los vasos es mediano mientras que para $E$. capuli y $E$. mayana es moderadamente pequeño; el parénquima axial en las cuatro especies se presenta en bandas, E. capuli presenta además vasicéntrico y difuso y $E$. mayana difuso escaso. En las especies de Veracruz los rayos son moderadamente numerosos $(\bar{x}=7)$ y en las estudiadas son pocos $(\bar{x}=3$ y 4); las fibras en las cuatro especies son libriformes con paredes gruesas y puntuaciones simples, E. mayana presenta gomas (Figura 4).

La presencia de poros pequeños, fibras con paredes gruesas y contenidos (gomas y cristales romboidales) refleja una estructura compacta, cualidades que permiten entender el uso de estas dos especies para la elaboración de durmientes $y$, en el caso de postes y pilares para construcción rural estas maderas utilizadas sin ningún tratamiento químico, podrían reflejar una resistencia natural al biodeterioro.

Por otra parte, según los datos del inventario forestal del ejido Cafetal Limones estas especies tienen buena presencia en la selva mediana subperennifolia (OEPFZM S.C., 2000), que en conjunto con otras especies mal llamadas corrientes y duras ya reportadas (Rebollar y Quintanar, 1998; 2000) cuya madera presenta similares cualidades anatómicas, estéticas y por las cualidades que los campesinos mayas les han conferido de resistencia y dureza para ser usadas en construcción rural pueden considerarse como especies alternativas para diversos usos comerciales. 


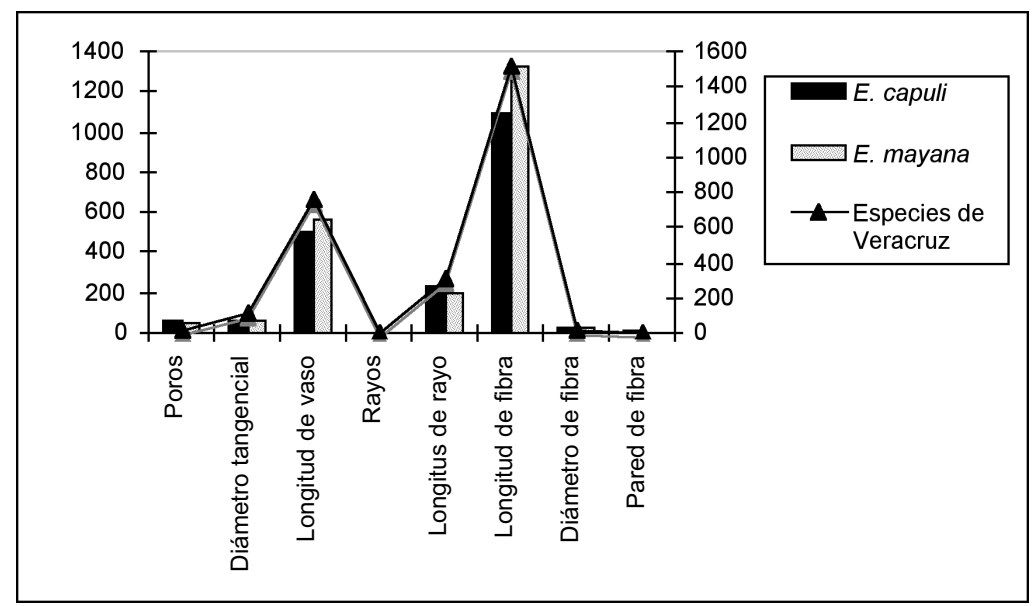

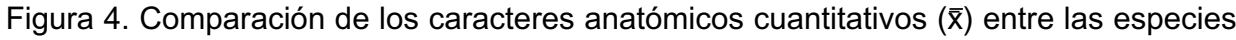
de Veracruz y las de Quintana Roo.

Es importante considerar este tipo de estudios que permiten implementar el conocimiento de la variedad de especies que manejan los ejidatarios como recurso de subsistencia que, en el caso de la actividad de producción de durmientes, les sigue generando un recurso económico confiable, ya que se continúa solicitando la compra del durmiente por algunas empresas privadas (OEPFZM S.C., 2000). Un factor importante que favorece este tipo de trabajos es la actitud abierta y positiva de los productores forestales de esta localidad quienes están interesados en mejorar sus prácticas agroforestales que eviten en lo posible el deterioro de sus selvas, ya que normalmente con sus programas de manejo forestal tienen una buena planeación para la producción tanto de madera como de otros recursos (frutales, hortalizas, agricultura de temporal).

\section{RECONOCIMIENTOS}

Las autoras agradecen el apoyo de los pobladores del ejido Cafetal Limones, en particular al comisario ejidal Carlos Torres, las facilidades brindadas en el trabajo de campo por compartir sus conocimientos de usos locales y nombres comunes de las especies colectadas y al señor Dionisio Yam Moo, su ayuda en la colecta de las especies. Al doctor Rafael Durán y a la doctora Ingrid Olmested (qepd), por la identificación de las muestras de herbario. A la doctora Carmen de la Paz Pérez-Olvera por la revisión y valiosas sugerencias al manuscrito, al biólogo Miguel Bravo Rivera, por su apoyo en la elaboración del mapa y a Ariadna Nicté Ramírez Ayala por la toma de fotografías.

\section{REFERENCIAS}

Anderson, E., J. Cauch, A. Dzih, S. Flores, G. Islebe, F. Medina, O. Sánchez y P. Valdez. 2005. Las plantas de los mayas: Etnobotánica en Quintana Roo, México. Comisión nacional para el conocimiento y uso de la biodiversidad y Colegio de la Frontera Sur. 206 p.

Ángeles, P. 1981. Anatomía de la madera de veintiún especies de la región de Los Tuxtlas, Veracruz. Tesis de licenciatura. Facultad de Ciencias, UNAM. $106 \mathrm{p}$. 
Arellano, J., S. Flores, J. Tun y M. Cruz. 2003. Nomenclatura, forma de vida, uso, manejo y distribución de las especies vegetales de la Península de Yucatán. Etnoflora Yucatanense. Fascículo 20. Facultad de Medicina Veterinaria y Zootecnia, UAY. $815 \mathrm{p}$.

Barajas-Morales, J., G. Ángeles y P. Solís. 1997. Anatomía de maderas de México: especies de una selva alta perennifolia I. Publicaciones especiales 16. Instituto de Biología, UNAM. 126 p.

Barrera, A., A. Barrera y R. López. 1976. Nomenclatura etnobotánica maya. Una interpretación taxonómica. Instituto Nacional de Antropología e Historia. Secretaría de Educación Pública. México. 238 p.

Cabrera, E., S. Torres y A. Curtis. 2001. Catálogo de árboles del jardín botánico "Dr. Alfredo Barrera Marín". Puerto Morelos, Quintana Roo, México. Ecosur. 111 p. (Manuscrito)

Cartujano, S., S. Zamudio, O. Alcántara e I. Luna. 2002. El bosque mesófilo de montaña en el municipio de Landa Matamoros, Querétaro, México. Boletín de la Sociedad Botánica de México 70:13-43.

Castillo, G., R. Robles y M. Medina. 2003. Flora y vegetación de la Sierra Cruz Tetela, Veracruz, México. Polibotánica 15:41-87.

Chattaway, M. 1932. Proposed standards for numerical value used in describing woods. Tropical Woods 29:20-28.

Gutiérrez, C. 2000. Listado florístico actualizado del estado de Campeche, México. Centro de Investigaciones Históricas y Sociales. Universidad Autónoma de Campeche. 95 p.

Gutiérrez, C. 2004. Listado florístico del norte de Chiapas: Catazajá y límites con Palenque, México. Polibotánica 17:107-124.

IAWA Committee. 1937. Standard terms of length of vessel members and wood fibers. Tropical Woods 51. $21 \mathrm{p}$.

IAWA Committee. 1939. Standard terms of size for vessel diameter and ray width. Tropical Woods 59:51-52.

IAWA Committee. 1989. IAWA list of microscopic features for hardwood identification. IAWA Bulletin 10:219-332.

Ibarra-Manríquez, G., J.L.R. Villaseñor y R. Durán García. 1995. Riqueza y endemismo del componente arbóreo de la Península de Yucatán, México. Boletín de la Sociedad Botánica de México 57:49-77.

INEGI. (Instituto Nacional de Estadística y Geografia). 2005. II Conteo de población y vivienda. Quintana Roo. http://www.inegi.gob.mx/est/ contenidos/espanolsistemas/conteo2005/ter2005/se lentcampo.aspx (21/mayo/2008).

Johnson, L.A.S. y B.G. Briggs. 1984. Myrtales and Myrtaceae a phylogenetic analysis. Annals of the Missouri Botanical Garden 71:700-756.

Kribs, D. 1968. Commercial foreign woods on the american market. Dover, Nueva York. 241 p.

Marchiori, J.N.C. 1984. Anatomia da madeira de Eugenia involucrate DC. (Myrtaceae). Ciencia e Natura, Santa Maria 6:127-136.

Martínez, E., M. Sousa y C. Ramos. 2001. Listado florístico de México. XXII Región de Calakmul, Campeche. México. Instituto de Biología. Universidad Nacional Autónoma de México. 55 p.

McVaugh, R. 1963. Flora of Guatemala Myrtaceae. Fieldiana: Botany. Chicago 
Natural History Museum Press 24:309373.

Merino, L. 1992. La experiencia en la organización forestal de la zona maya. México. El Cotidiano 48:41-43.

Monroy-Ortiz, C. y R. Monroy. 2006. Las plantas, compañeras de siempre: la experiencia en Morelos. Comisión nacional para el conocimiento y uso de la biodiversidad. México. 582 p.

Munsell Color Company. 1954. Munsell soil color charts. Baltimore, Maryland. $17 \mathrm{p}$.

OEPFZM, S.C. (Organización de Ejidos Productores Forestales de la Zona Maya, S.C.) 2000. Dirección Técnica Forestal. Informe de paso de año 2000 del ejido Cafetal Limones. Felipe Carrillo Puerto, Quintana Roo. México.

Padilla-Velarde, E., R. Cuevas-Guzmán, G. lbarra-Manríquez y S. Moreno-Gómez. 2006. Riqueza y biogeografía de la flora arbórea del estado de Colima, México. Revista Mexicana de Biodiversidad 77:271-295.

Ramos, C. y V. Díaz. 1981. Instrucciones para colectar muestras de madera para estudios tecnológicos. Boletín divulgativo del Instituto Nacional de Investigaciones Forestales. México. No. 54. 15p.

Rebollar, S., C. de la Paz Pérez-O. y A. Quintanar. 1987. Maderas de la Península de Yucatán. México. Biótica 12:159-179.

Rebollar, S., C. de la Paz Pérez-O. y A. Quintanar. 1993. Anatomía de la madera de cinco especies de Quintana
Roo, México. Boletín de la Sociedad Botánica de México 53:103-124.

Rebollar, S. y A. Quintanar. 1998. Anatomía y usos de la madera de ocho especies tropicales de Quintana Roo, México. Revista de Biología Tropical 46(4):1047-1057.

Rebollar, S. y A. Quintanar. 2000. Anatomía y usos de la madera de siete árboles tropicales de México. Revista de Biología Tropical 48(2/3):596-578.

Record, S. y R. Hess. 1949. Timbers of the new world. New Haven Yale University Press, London. 639 p.

Sosa, V., S. Flores, V. Rico-Gray, R. Lira y J. Ortiz. 1985. Lista florística y sinonimia maya. Etnoflora Yucatanense. Fascículo I. Instituto Nacional de Investigaciones sobre Recursos Bióticos. Xalapa, Veracruz. 225 p.

Terán, S. y Ch. Rasmussen. 1994. La milpa de los mayas. La agricultura de los mayas prehispánicos y actuales en el noreste de Yucatán. Gobierno del estado de Yucatán y gobierno de Dinamarca. Mérida, Yucatán. México. 349 p.

Tortorelli, L. 1956. Maderas y bosques argentinos. ACME, Buenos Aires. 910 p.

Valiente-Banuet, A., F. González y D. Piñero. 1995. La vegetación selvática de la región de Gómez Farías, Tamaulipas, México. Acta Botánica de México 3:1-36.

Yáñez, L. 2004. Las principales familias de árboles en México. Universidad Autónoma Chapingo. División de Ciencias Forestales. Texcoco, Estado de México. $189 \mathrm{p}$.

Manuscrito recibido el 12 de agosto de 2008

Aceptado el 11 de diciembre de 2009

Este documento se debe citar como: Rebollar-Domínguez, S. y N.A. Tapia-Torres.2010. Anatomía de la madera de dos especies de Eugenia (Myrtaceae) de Quintana Roo, México. Madera y Bosques 16(1):85-98. 